\title{
DISTRIBUCIÓN ESPACIAL Y CUANTIFICACIÓN DE LOS SERVICIOS Y CONTRA- SERVICIOS ECOSISTÉMICOS DE LA AGRICULTURA EN LA REGION DE MURCIA (PÓSTER)
}

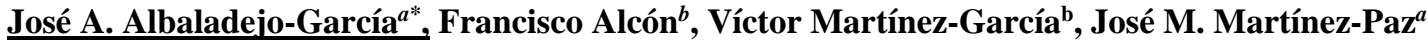

a Departamento de Economía Aplicada. Universidad de Murcia (Murcia, joseantonio.albaladejo@um.es, jmpaz@um.es).

b ETSIA. Universidad Politécnica de Cartagena (Cartagena, francisco.alcon@upct.es, victor.martinez@upct.es).

\begin{abstract}
Resumen
Uno de los retos del estudio integral de los agroecosistemas es la determinación de los servicios y contra servicios ecosistémicos de la agricultura (SyCSEA), valorando su provisión y relacionándola con las características del sistema productivo. Dada la importancia de los beneficios y perjuicios que los SyCSEA proporcionan al bienestar humano, comprender sus relaciones es crucial para una gestión óptima de la agricultura. El objetivo de este trabajo es cuantificar la provisión y distribución espacial de los SyCSEA en la Región de Murcia, en una zona semiárida donde coexiste la agricultura de secano con regadíos tradicionales y otros muy tecnificados. Para este análisis se seleccionaron, en primer lugar, los seis SyCSEA más importantes, a juicio de expertos de la zona: provisión de alimentos, consumo de agua, regulación climática, contaminación de acuíferos, recreación y biodiversidad. Estos SyCSEA se cuantificaron combinando datos estadísticos y de Sistemas de Información Geográfica. Los resultados pusieron de manifiesto la heterogeneidad espacial en la provisión de SyCSEA en la Región, pudiendo destacar del análisis de correlación, entre otros, la sinergia del consumo de agua con la provisión de alimentos o el tradeoff entre la contaminación de acuíferos y la recreación.
\end{abstract}

Palabras clave: Análisis espacial, SIG, provisión, SE-España.

\section{Introducción}

Los agroecosistemas mediterráneos se caracterizan por una prolongada influencia antrópica que ha dado lugar a una elevada variedad de provisión de servicios y contra servicios ecosistémicos de la agricultura (SyCSEA). Estos se corresponden con los beneficios y perjuicios que las personas obtienen de los agroecosistemas (Balzan et al., 2020) y pueden agruparse en cuatro categorías: abastecimiento, regulación, culturales y de soporte.

El análisis de los SyCSEA en su conjunto permite reflejar de forma clara la multifuncionalidad de estos ecosistemas, mejorando la información para su gobernanza y disminuyendo el riesgo de fracaso de las políticas agrarias (Turkelboom et al., 2018).

Si bien en agroecosistemas mediterráneos se ha venido analizado la demanda social de SyCSEA (Zabala et al., 2021), no son tan abundantes los estudios que presenten la provisión de estos servicios. En este sentido, el objetivo de este trabajo ha sido cuantificar la provisión y distribución espacial de los SyCSEA en la Región de Murcia, área mediterránea semiárida en la cual convive un modelo de cultivos de secano, regadíos tradicionales y zona regables muy tecnificadas.

\section{Materiales y métodos}

Para la cuantificación de los SyCSEA se han utilizado herramientas de Sistemas de Información Geográfica (SIG), con el apoyo de la información secundaria de las estadísticas oficiales. Asimismo, se ha realizado un análisis multicriterio para conocer los niveles de provisión en cada municipio, y análisis de correlación de Pearson con los que determinar las sinergias y trade-offs.

A partir de una consulta a expertos implicados en la gestión agroambiental de la Región de Murcia(Zabala et al., 2021), se han seleccionado los 6 SyCSEA de los 15 que fueron identificados en la literatura que podían influir en el bienestar de la población. Estos servicios y contra servicios fueron: 2 servicios de abastecimiento (provisión alimentos y consumo de agua), 3 servicios de regulación (regulación climática, contaminación de acuíferos y biodiversidad) y 1 servicio cultural (recreación). En el Cuadro 1 se recogen los SyCSEA utilizados, así como los indicadores asociados a cada SyCSEA, los cuales han sido agregados a las superficies agrícolas de cada uno de los 45 municipios de la Región de Murcia. 
Cuadro 1. Indicadores utilizados para cuantificar los SyCSEA en la Región de Murcia

\begin{tabular}{|c|c|c|c|c|}
\hline SyCSEA & Indicador & Unidades & Variables utilizadas & Fuente \\
\hline \multicolumn{5}{|c|}{ Abastecimiento } \\
\hline $\begin{array}{l}\text { Provisión alimentos } \\
\text { (INGR) }\end{array}$ & $\begin{array}{l}\text { Ingresos anuales } \\
\text { percibidos por los } \\
\text { agricultores }\end{array}$ & $€ /$ ha año & $\begin{array}{l}\text {-Rendimientos } \\
\text {-Precios } \\
\text {-Superficies cultivos en municipios }\end{array}$ & $\begin{array}{l}\text {-Anuarios } \\
\text { estadísticos } \\
\text {-SIG }\end{array}$ \\
\hline $\begin{array}{l}\text { Consumo agua } \\
\text { (CONSAG) }\end{array}$ & $\begin{array}{l}\text { Consumo de agua } \\
\text { para el regadío }\end{array}$ & $\mathrm{m}^{3} / \mathrm{ha}$ año & $\begin{array}{l}\text {-Consumo agua cultivos } \\
\text {-Superficies cultivos en municipios }\end{array}$ & $\begin{array}{l}\text {-Anuarios } \\
\text { estadísticos } \\
\text {-SIG }\end{array}$ \\
\hline \multicolumn{5}{|c|}{ Regulación } \\
\hline $\begin{array}{l}\text { Regulación climática } \\
\text { (REGCLIM) }\end{array}$ & $\begin{array}{l}\text { Reducción de la } \\
\text { temperatura } \\
\text { superficial terrestre } \\
\end{array}$ & $\begin{array}{l}\text { Índice } \\
0-1\end{array}$ & $\begin{array}{l}\text {-Temperatura superficie terrestre } \\
\text {-NDVI, NDWI y Altitudes } \\
\text {-Superficies cultivos en municipios }\end{array}$ & $-\mathrm{SIG}$ \\
\hline $\begin{array}{l}\text { Contaminación } \\
\text { acuíferos } \\
\text { (CONTACU) }\end{array}$ & $\begin{array}{l}\text { Contaminación de } \\
\text { las aguas } \\
\text { subterráneas }\end{array}$ & $\begin{array}{l}\text { Índice } \\
0-1\end{array}$ & $\begin{array}{l}\text {-Zonas vulnerables y no vulnerables } \\
\text {-Impacto cultivos en acuíferos } \\
\text {-Superficies cultivos en municipios }\end{array}$ & $\begin{array}{l}\text { - Anuarios } \\
\text { estadísticos } \\
\text {-SIG }\end{array}$ \\
\hline $\begin{array}{l}\text { Biodiversidad } \\
\quad(\mathrm{BIO})\end{array}$ & $\begin{array}{l}\text { Riqueza de especies } \\
\text { de aves }\end{array}$ & $\begin{array}{l}\text { Índice } \\
0-1\end{array}$ & $\begin{array}{l}\text {-Presencia edificaciones, presencia } \\
\text { patrimonio hidráulico, proximidad } \\
\text { carretera, presencia zona ZEPA } \\
\text {-Superficies cultivos en municipios }\end{array}$ & -SIG \\
\hline \multicolumn{5}{|c|}{ Cultural } \\
\hline $\begin{array}{l}\text { Recreación } \\
\text { (RECRE) }\end{array}$ & $\begin{array}{l}\text { Oportunidad disfrute } \\
\text { actividades } \\
\text { recreativas }\end{array}$ & $\begin{array}{l}\text { Índice } \\
0-1\end{array}$ & $\begin{array}{l}\text {-NDVI, tipo de porte, tipo de riego, } \\
\text { presencia edificaciones, presencia } \\
\text { patrimonio hidráulico, proximidad } \\
\text { carretera, presencia diversidad } \\
\text { vegetal, ubicación cultivos en } \\
\text { espacios naturales protegidos } \\
\text {-Superficies cultivos en municipios }\end{array}$ & -SIG \\
\hline
\end{tabular}

Fuente: elaboración propia

La superficie agrícola cultivada de la Región de Murcia es en la actualidad de unas 312.131 ha, de las cuales el 50,61\% corresponde a cultivos en regadío, y el resto a secano (CREM, 2019). Se han utilizado las superficies (parcelas) agrícolas declaradas por los agricultores en el SIGPAC formadas por cultivos de cítricos, frutales, hortícolas, olivares, y viñedos en regadío, y por cultivos de frutales, herbáceos, olivares y viñedos en secano. También se incluye un uso del suelo específico, denominado Huerta, el cual es una combinación de cítricos y hortícolas asociados a una agricultura de regadío tradicional, y que principalmente se localiza en torno a las vegas del río Segura. El nivel espacial de agregación utilizado en la presentación de resultados ha sido el de los 45 municipios que componen la Región, limite espacial en la disponibilidad de algunos de los datos utilizados.

\section{Resultados}

En el Mapa 1 se visualiza la distribución espacial de la provisión de los 6 tipos de SyCSEA para cada uno de los municipios de la Región de Murcia. Este mapa muestra una mayor provisión de los servicios y contra servicios de abastecimiento en municipios con una mayor superficie agrícola dedicada al regadío intensivo (costeros como Torre Pacheco, San Javier...), y, por el contrario, una menor provisión en municipios donde predominan los cultivos de secano (Noroeste como Moratalla, Caravaca de la Cruz...).

En cuanto a los servicios de regulación, el índice de regulación climática, elaborado a partir de AlbaladejoGarcía et al., (2020), muestra unas temperaturas menores en municipios costeros y con cultivos predominantes de regadío (ej: Cartagena, Águilas...). Asimismo, el índice de contaminación de acuíferos, correspondiente a un contra-servicio de regulación, ha puesto de relieve el impacto negativo que tiene la agricultura intensiva en algunos municipios del Campo de Cartagena y el Campo de Lorca en las aguas subterráneas. Respecto al tercer servicio de regulación, la biodiversidad, se aprecia que en los municipios por donde discurre el río Segura, y que coincide con las superficies de huerta tradicional, es donde se dan los mayores valores del índice de riqueza de aves. Por el contrario, la menor biodiversidad se ha dado en municipios donde mayoritariamente se desarrolla una agricultura intensiva (ej: Campo de Cartagena). 
Por último, en cuanto al servicio cultural de la recreación, el índice elaborado ha mostrado un patrón espacial similar al de la biodiversidad, destacando el alto valor recreativo de municipios como Cieza y Ricote, entre otros.

Mapa 1. Distribución espacial de la provisión de SyCSEA en la Región de Murcia
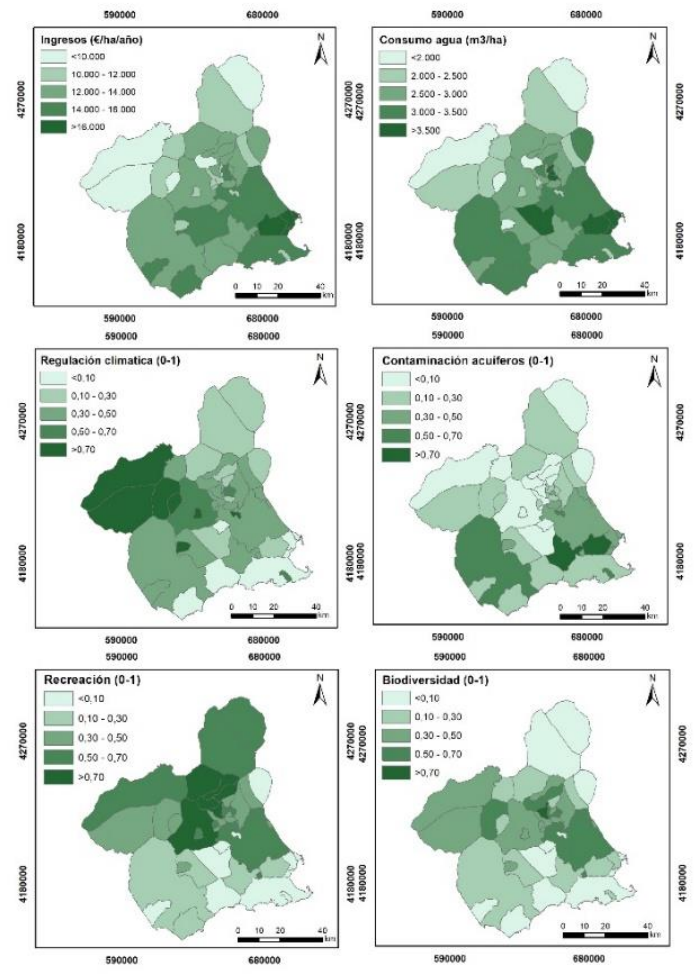

Fuente: elaboración propia

Tras cuantificar y representar espacialmente la provisión de los 6 tipos de SyCSEA se ha llevado a cabo un análisis multicriterio para determinar los municipios que cuentan con diferentes niveles de provisión general de SyCSEA (Mapa 2).

En este análisis se han utilizado dos sistemas de ponderaciones para agregar los SyCSEA, una vez normalizados:

I) Considerando que todos los SyCSEA tienen la misma importancia.

II) Considerando la importancia relativa de cada servicio recogidas en el trabajo de Zabala et al. (2021): 0,05 INGR; 0,15 CONSAG; 0,15 CONTACU; 0,07 REGCLIM; 0,38 BIO у 0,20 RECRE.

Para ambas estimaciones (con y sin ponderaciones) se observó que en municipios con grandes superficies de huerta tradicional y de secano (Ojós, Cieza, Blanca, Abarán, Villanueva del Segura y Ricote) es donde se da una mayor provisión de SyCSEA. Por el contrario, en los municipios donde predomina una agricultura intensiva de hortícolas y cítricos (Fuente Álamo, Torre Pacheco y Alcantarilla) tiene lugar la menor provisión de los SyCSEA.

Mapa 2. Niveles de provisión de SyCSEA en la Región de Murcia: (a) sin ponderaciones (b) con ponderaciones

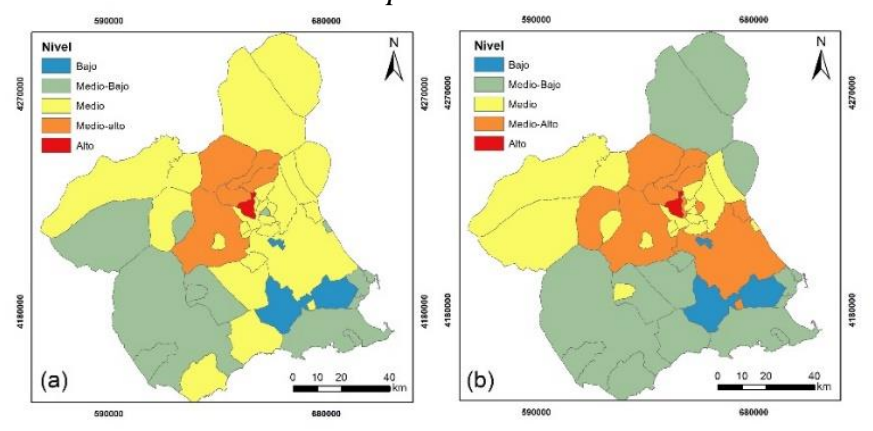

Fuente: elaboración propia 
Finalmente, se ha realizado un análisis de correlación lineal entre los valores de los índices de los 6 SyCSEA (Cuadro 2). Existen correlaciones positivas entre, por un lado, los ingresos, el consumo de agua y la contaminación de acuíferos, y por otro, entre la biodiversidad y la recreación. Las correlaciones negativas se producen entre la reducción de temperaturas con los ingresos y el consumo de agua, observándose también una relación negativa entre la contaminación de acuíferos y las actividades recreativas.

Cuadro 2. Correlaciones de Pearson entre los diferentes SyCSEA

\begin{tabular}{|l|c|c|c|c|c|c|}
\hline Variables & INGR & CONSAG & REGCLIM & CONTACU & RECRE & BIO \\
\hline INGR & 1 & & & & & \\
\hline CONSAG & $0,94 * * *$ & 1 & & & & \\
\hline REGCLIM & $-0,56^{* * *}$ & $-0,57^{* * *}$ & 1 & & & \\
\hline CONTACU & $0,27^{*}$ & 0,17 & $-0,01$ & 1 & & \\
\hline RECRE & $-0,22$ & $-0,15$ & $-0,08$ & $-0,23 *$ & 1 & \\
\hline BIO & $-0,16$ & $-0,03$ & 0,15 & $-0,18$ & $0,37 * *$ & 1 \\
\hline
\end{tabular}

Fuente: elaboración propia. $\left({ }^{*} p<0,10 ; * * p<0,05 ; * * * p<0,01\right)$. Los colores rojo y azul indican correlaciones positivas y negativas, respectivamente.

\section{Conclusiones}

En este trabajo ha puesto de manifiesto la heterogeneidad espacial en la provisión de SyCSEA en la Región de Murcia a partir de un análisis territorial a nivel municipal. Los municipios que cuentan con una superficie agrícola mayoritariamente de regadío tradicional y de secano son los que muestran una mayor provisión global de los SyCSEA, y, por tanto, son las zonas donde destaca el carácter multifuncional de la agricultura, proporcionando bienes y servicios que van más allá de la tradicional función de producción de alimentos. En cambio, municipios con extensas superficies de una agricultura de regadío intensiva, a pesar de ser altamente productivas, tienen una menor provisión global de SyCSEA. En este sentido, a la hora de contemplar rentas complementarias a los agricultores, como las que procedan de la Política Agraria Común, éstas quedarían justificadas en aquellas zonas donde tiene lugar la provisión conjunta tanto de bienes públicos a la sociedad como de bienes privados, de forma que se favorecieran, entre otros, la biodiversidad o el disfrute de un ecosistema que puede ser atractivo por muchos ciudadanos, como por ejemplo lo son las zonas de la "Huerta de Murcia" y el "Valle de Ricote".

El análisis de correlaciones ha mostrado sinergias entre la biodiversidad y las actividades recreativas, siendo en agroecosistemas menos tecnificados donde se fomenta la provisión de estos dos servicios. A su vez, cabe mencionar el efecto que tiene el regadío, y el consumo de agua asociado, en la reducción de temperaturas y en el aumento de los ingresos de los agricultores, que lleva a su vez asociado la contaminación de aguas subterráneas. Estos resultados muestran la necesidad de integrar el análisis conjunto de los SyCSEA en los procesos de la toma de decisiones y adaptar el marco de gobernanza de los agroecosistemas a la provisión y preferencias sociales de los mismos.

\section{Agradecimientos}

Este trabajo se ha realizado en el marco del proyecto 20912/PI/18 financiado por la "Fundación SénecaAgencia de Ciencia y Tecnología de la Región de Murcia” y por el MECD (FPU16/03562).

\section{Bibliografía}

Albaladejo-García, J. A., Alcon, F., \& Martínez-Paz, J. M. (2020). "The irrigation cooling effect as a climate regulation service of agroecosystems". Water, 12(6), 1553.

Balzan, M. V., Sadula, R., \& Scalvenzi, L. (2020). “Assessing ecosystem services supplied by agroecosystems in Mediterranean Europe: A literature review”. Land, 9(8), 245.

CREM (2019). Evolución de la superficie de las tierras de cultivo según municipios, por tipo de cultivo. https://econet.carm.es/inicio/-/crem/sicrem/PU_datosBasicos/sec49.html

Turkelboom, F., Leone, M., Jacobs, S., Kelemen, E., García-Llorente, M., Baró, F., \& Rusch, V. (2018). "When we cannot have it all: Ecosystem services trade-offs in the context of spatial planning”. Ecosystem services, 29, 566-578.

Zabala, J. A., Martínez-Paz, J. M., \& Alcon, F. (2021). “A comprehensive approach for agroecosystem services and disservices valuation". Science of The Total Environment, 768, 144859. 